\title{
The effects of optimizing blood inflow in the pedicle on perforator flap survival: A pilot study in a rat model
}

\author{
Radu Olariu ${ }^{1}$, Helen Laura Moser ${ }^{1}$, Ioana Lese ${ }^{1}$, Dan Sabau ${ }^{2}$, Alexandru Valentin Georgescu ${ }^{3}$, \\ Adriaan Ockert Grobbelaar ${ }^{1}$, Mihai Adrian Constantinescu ${ }^{1}$ \\ ${ }^{1}$ Department of Plastic and Hand Surgery, Inselspital University Hospital Bern, Bern, Switzerland; ${ }^{2}$ Surgical Clinic 2, Emergency County \\ Hospital, Victor Papilian Faculty of Medicine, Lucian Blaga University of Sibiu, Sibiu; ${ }^{3}$ Department of Plastic Surgery, Rehabilitation \\ Hospital, Iuliu Hatieganu University of Medicine and Pharmacy, Cluj-Napoca, Romania
}

Background Perforator flaps have led to a revolution in reconstructive surgery by reducing donor site morbidity. However, many surgeons have witnessed partial flap necrosis. Experimental methods to increase inflow have relied on adding a separate pedicle to the flap. The aim of our study was to experimentally determine whether increasing blood flow in the perforator pedicle itself could benefit flap survival.

Methods In 30 male Lewis rats, an extended posterior thigh perforator flap was elevated and the pedicle was dissected to its origin from the femoral vessels. The rats were assigned to three groups: control (group I), acute inflow (group II) and arterial preconditioning (group III) depending on the timing of ligation of the femoral artery distal to the site of pedicle emergence. Digital planimetry was performed on postoperative day (POD) 7 and all flaps were monitored using laser Doppler flowmetry perioperatively and postoperatively in three regions (P1-proximal flap, P2-middle of the flap, P3-distal flap).

Results Digital planimetry showed the highest area of survival in group II (78.12\% $\pm 8.38 \%)$, followed by groups III and I. The laser Doppler results showed statistically significant higher values in group II on POD 7 for P2 and P3. At P3, only group II recorded an increase in the flow on POD 7 in comparison to POD 1.

Conclusions Optimization of arterial inflow, regardless if performed acutely or as preconditioning, led to increased flap survival in a rat perforator flap model.

Keywords Surgery, plastic / Perforator flap / Animal experimentation
Correspondence: Radu Olariu Department of Plastic and Hand Surgery, Inselspital University Hospital Bern, University of Bern, Freiburgstrasse, $\mathrm{CH}-3010$ Bern, Switzerland Tel: +41-31-632-80-14 Fax: +41-31-632-12-28 E-mail: radu.olariu@insel.ch

This work was funded by the Department of Plastic and Hand Surgery, Inselspital University Hospital Bern, Bern, Switzerland.

This work will form part of the PhD thesis of Radu Olariu at the Lucian Blaga University of Sibiu, Sibiu, Romania.

\section{INTRODUCTION}

Increasing knowledge of the perforator vessel anatomy and skin perfusion has enabled clinicians to use the perforator flap technique in reconstructions after various defects [1]. This tech- nique was pioneered by Koshima and Soeda [2], who marked the beginning of the perforator flap era in 1989 when they described the first inferior epigastric artery skin flap without rectus abdominis muscle, thereby reducing the associated morbidity at the donor site. Since then, major efforts have been made to im- 
prove the outcomes of perforator flaps and thereby to widen the indications for this surgical technique, including, in recent years, the development of local pedicled perforator flaps [3] that do not require microsurgical anastomoses. Although they have the advantages of reducing morbidity and overall operating time [4], local perforator flaps seem to have a higher rate of partial necrosis [5], which has prompted researchers and clinicians to search for methods of increasing perforator flap survival.

Vessel manipulation, whether aimed at increasing inflow or optimizing outflow, has been frequently employed by researchers in order to influence the survival of perforator flaps, with largely inconclusive results [6-10]. In all models, inflow and/or outflow manipulations were performed by selectively adding new vascular sources, a procedure that is often difficult to use in clinical situations. From a clinical point of view, the main advantages of local perforator flaps are the rapidity of the procedure (compared to free tissue transfer), reconstruction with tissues located near the defect ("like with like"), and the absence of any need for microvascular surgery [11]. Efforts to improve the reliability of perforator flaps should preserve these advantages. Therefore, any vascular manipulations should aim not to limit flap transposition and not to add a microvascular procedure. However, experimental studies with the goal of increasing flow in the pedicle of the flap are lacking. Our study was conducted to address this lack of experimental data, and therefore aimed to evaluate the effect of optimizing arterial inflow in the pedicle itself on flap perfusion and survival in a rat musculocutaneous perforator flap model.

\section{METHODS}

Thirty Lewis rats weighing 250-300 g were used for this study. All animals were treated according to the Public Health Service Policy on Humane Care and Use of Laboratory Animals during the entire experiment. They were provided with standard laboratory food and water ad libitum throughout the entire observation period and their health status was assessed daily. This study was approved by the Ethics Committee for Animal Experimentation, Bern, Switzerland (approval No. 89/16).

Based on the model presented by Coskunfirat et al. [12], we elevated a posterior thigh perforator flap in all animals and dissected the musculocutaneous perforator up to its emergence from the femoral vessels in all rats. We randomly assigned the rats to the following experimental groups based on how the vascular pedicle was manipulated, as follows: (1) Control ( $n=10)$ : no vascular manipulation, flap was sutured back in its original position; (2) Acute inflow optimization $(\mathrm{n}=10)$ : the femoral artery was ligated just distal to the emergence of the perforator at the time of flap raising, and the flap was then sutured back in its original position; (3) Arterial preconditioning $(\mathrm{n}=10)$ : ligation of the femoral artery distal to the emergence of the perforator was performed through inguinal crease incision 5 days before flap raising. Five days later the flap was raised as described below and sutured back in its original position.

All procedures were performed under continuous inhalation anesthesia. To induce anesthesia, rats were placed in an induction chamber, where $5 \%$ isoflurane with oxygen $(1 \mathrm{~L} / \mathrm{min})$ was administered for 2-3 minutes. Maintenance anesthesia was then administered using $1 \%-1.5 \%$ isoflurane with $0.6 \mathrm{~L} / \mathrm{min}$ oxygen. A normal body temperature was maintained through thermal pads, and the rats were treated with ophthalmic ointment in both eyes to prevent desiccation. Preemptive analgesia was applied through a subcutaneous injection of buprenorphine $(50 \mu \mathrm{g} / \mathrm{kg})$ 30 minutes preoperatively and postoperatively as needed according to a standardized animal welfare checklist.

\section{Surgical technique of flap raising}

The right half of the rats' dorsum was shaved and operative markings were drawn in a similar manner for all animals, with a rectangle measuring $9 \mathrm{~cm}$ in length and $3 \mathrm{~cm}$ in width, beginning at a line uniting the knee joint anteriorly and the ischial tuberosity posteriorly. The midline of the rectangle was drawn, along with the following three points: $\mathrm{P} 1,2 \mathrm{~cm}$ cranial from the caudal border (approximately overlying the perforator); P2, 3 $\mathrm{cm}$ cranial from P1; P3, $3 \mathrm{~cm}$ cranial from P2.

After incising the entire flap, a retrograde flap elevation underneath the panniculus carnosus was performed until the posterior thigh perforator, arising through the biceps femoris muscle, was identified. It was then carefully dissected, leaving a muscle cuff around the perforator to ensure a complication-free dissection. The perforator was followed until the femoral vessels were reached on the medial side of the thigh. An additional incision was placed in the inguinal crease to gain access to the femoral vessels, and the femoral artery was ligated distal to the perforator emergence. The femoral vein was not altered. The flap was then sutured back in place with Prolene 5-0 running sutures. In the arterial preconditioning group, an incision was made in the inguinal crease 5 days before raising the flap and the femoral artery was similarly ligated distal to the emergence of the perforator. Fig. 1 details the operative technique.

Just before closing the skin, the rats received buprenorphine subcutaneously $(50 \mu \mathrm{g} / \mathrm{kg}$ ). Analgesia (buprenorphine, $50 \mu \mathrm{g} / \mathrm{kg}$ subcutaneously) was administered postoperatively as needed (maximally every 12 hours) according to a standardized animal welfare checklist. The rats were observed for 7 days postoperatively, with daily assessments of their health status based on stan- 


\section{Fig. 1. Operative technique}

(A) Preoperative markings. (B) After the skin incision, the perforator arising through the biceps femoris muscle is identified. (C) Completed dissection of the perforator from the posterior side with a minimal protective muscle cuff. (D) Completion of dissection from the anterior aspect, with identification of the emergence of the perforator pedicle from the femoral vessels. (E) Inflow manipulation by ligation of the femoral artery distal to the perforator emergence. (F) Final image after resuturing the flap in its original position.
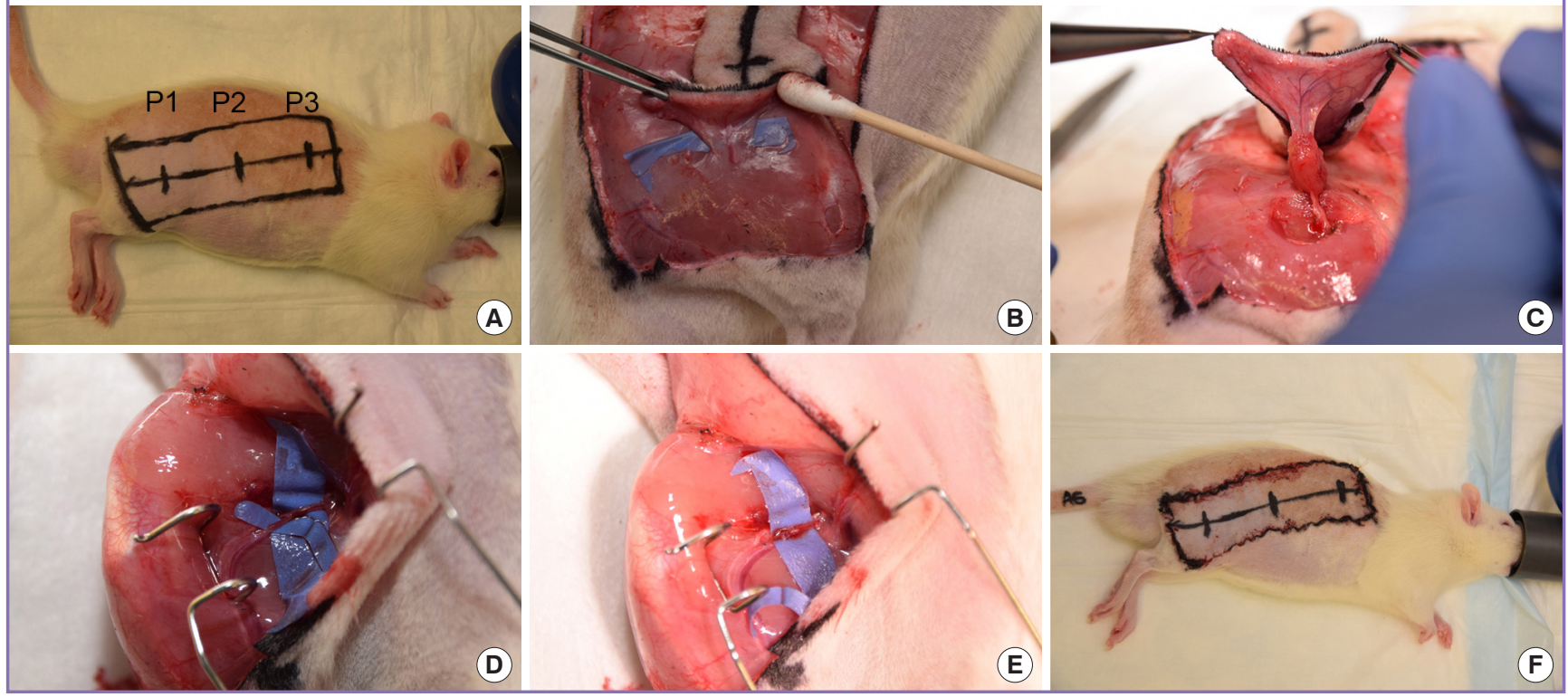

dard approved checklists. All rats were euthanized on postoperative day (POD) 7 by injecting $150 \mu \mathrm{g} / \mathrm{kg}$ pentobarbital intraperitoneally, and death was confirmed by bilateral thoracotomy.

\section{Flap assessment}

\section{Digital planimetry}

After the rats were euthanized, on POD 7, photographs were taken of the flaps from a distance of $40 \mathrm{~cm}$ using a Nikon D3300 camera with a Sigma 18-250 mm lens and a focal length of 75 $\mathrm{mm}$. The images were then processed with Adobe Photoshop (Adobe, San Jose, CA, USA). After the images were converted to BMP format, the pixel counts of the viable flap area (with relation to gray levels) and of the total flap area were counted, as has been described in the literature [13]. The flap viability area was then calculated using the following formula:

Flap viability area $=\frac{\text { viable area }(\text { number of pixels })}{\text { total area }(\text { number of pixels })} \times 100$

\section{Laser Doppler flowmetry}

Scanning laser Doppler flowmetry was performed using an Aimago EasyLDI Perfusion camera (Aïmago SA, Lausanne, Switzerland) on all the marked points (P1, P2, P3) before raising the flap, after raising the flap, immediately after completing the operation (postoperatively), and on POD 1 and 7. For group III, in which vessel manipulation was performed 5 days before rais- ing the flap, an additional reading took place just before vessel ligation. The results were expressed as mean perfusion units and P1 was taken as the reference point. The measured results for $\mathrm{P} 2$ and $\mathrm{P} 3$ were then expressed as percentages relative to $\mathrm{P} 1$.

\section{Statistical analysis}

Data analysis was performed with SPSS version 21.0 (IBM Corp., Armonk, NY, USA). The non-parametric Kruskal-Wallis test was conducted to compare blood flow among the three groups. Subsequently, pairwise comparisons were performed using the Dunn procedure with the Bonferroni correction for multiple comparisons. One-way analysis of variance and TukeyKramer post hoc analysis were used for the normally distributed digital planimetry measurements. The results were considered statistically significant at $\mathrm{P}<0.05$.

\section{RESULTS}

All animals survived to the experimental endpoint on POD 7 without any signs of functional impairment in all groups according to our checklists.

\section{Digital planimetry}

All flaps showed typical distal necrosis (Fig. 2). The flap survival areas were significantly different between the groups $(\mathrm{F}[2,26]=$ 29.28; $\mathrm{P}<0.001)$. Fig. 3 shows a graphical representation of the 


\section{Fig. 2. Typical appearance of flaps on POD 7}

(A) Group I (control). (B) Group II (acute inflow optimization). (C) Group III (arterial preconditioning). POD, postoperative day.
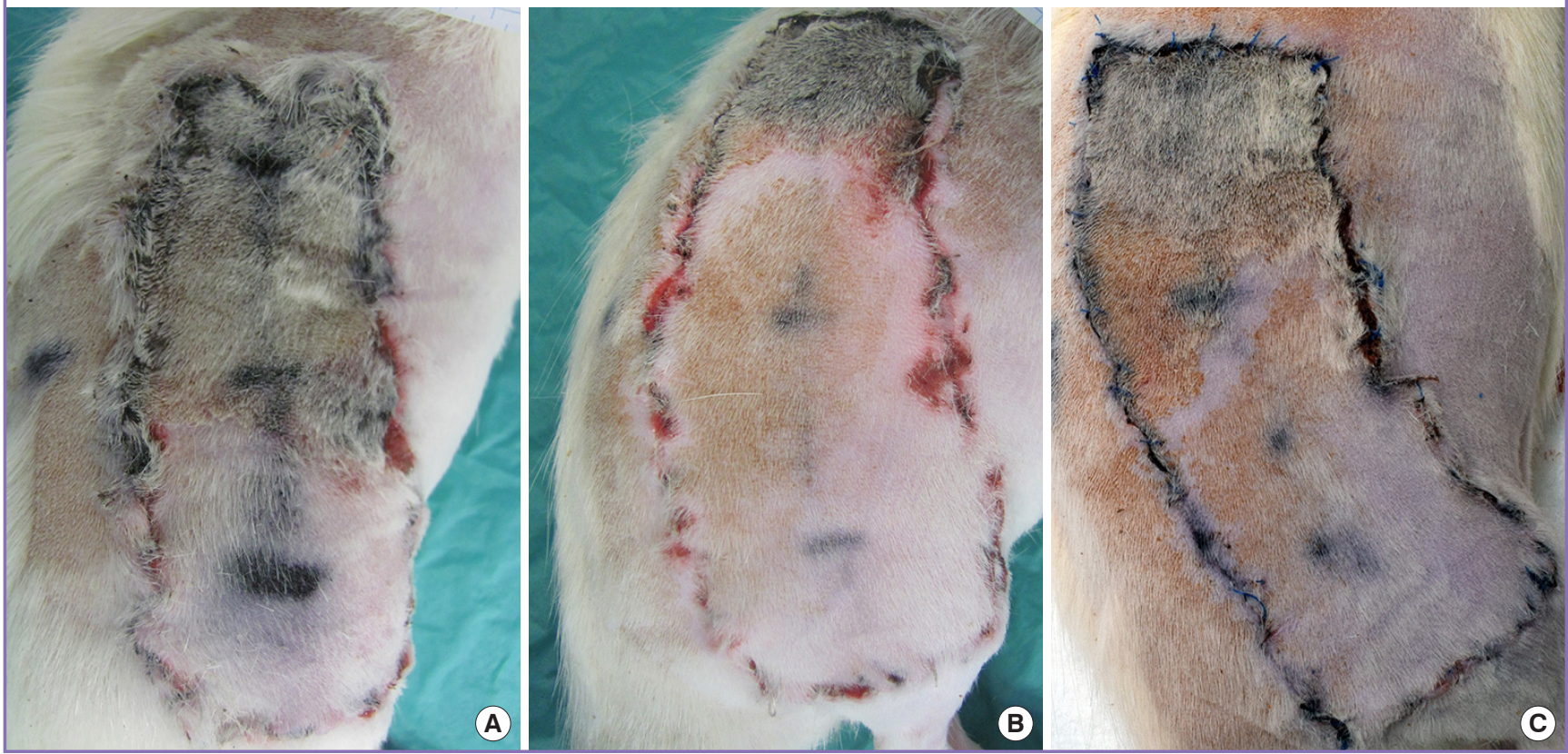

\section{Fig. 3. Digital planimetry: flap survival area}

Group I (control), group II (acute inflow optimization), and group III (arterial preconditioning). ${ }^{\text {a) }}$ Statistically significant differences $(\mathrm{P}<0.05)$.

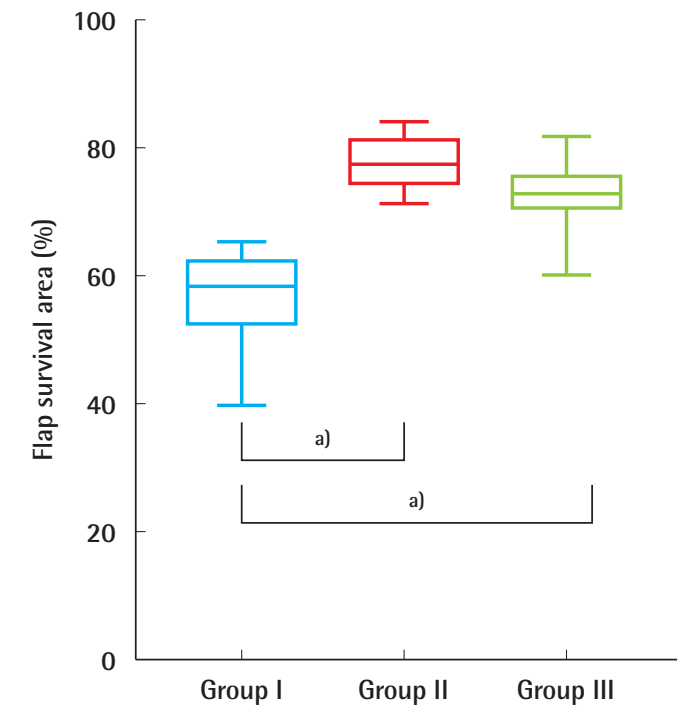

digital planimetry results. Data are presented as mean \pm standard deviation. The highest flap survival percentage was found in group II $(78.12 \% \pm 8.38 \%)$, followed by group III $(73.09 \% \pm 5.75 \%)$. The control group had the lowest values $(56.55 \% \pm 8.38 \%)$.

\section{Laser Doppler flowmetry}

The distribution of the blood flow was similar in all groups in all tests, as assessed by a visual inspection of the boxplots.

\section{Results for P2}

Fig. 4 shows the median percentage values obtained for P2 in all groups. The only statistically significant difference between groups was seen on POD 7, at which time group II had higher values than group $\mathrm{I}(\mathrm{P}=0.02)$.

The within-group analysis revealed a statistically significant decrease in the flow between the measurements taken before raising the flap and POD $1(\mathrm{P}<0.05)$ in all groups. The improvement of the flow between POD 1 and POD 7 was statistically significant in all groups $(\mathrm{P}<0.05)$. The acute inflow optimization group (group II) showed a particularly significant flow increase, from a median value of $85.49 \%$ to $135.8 \%$, from the preoperative measurements to POD 7.

\section{Results for $P 3$}

As depicted in Fig. 5, the flow decreased within all groups between the measurements taken after raising the flap and POD 7 $(\mathrm{P}<0.05)$. The difference in the flow between before the flap was raised or vessel manipulation was performed and POD 7 was statistically significant in all groups $(\mathrm{P}<0.05)$. On POD 7 , group II (acute inflow optimization) was the only group that recorded an increased flow in comparison to POD $1(\mathrm{P}<0.05)$. Furthermore, the flow in group II was significantly higher than 


\section{Fig. 4. Laser Doppler flowmetry: results for P2}

Median laser Doppler flowmetry results for P2 at different time points. Group I (control), group II (acute inflow optimization), and group III (arterial preconditioning). POD, postoperative day.

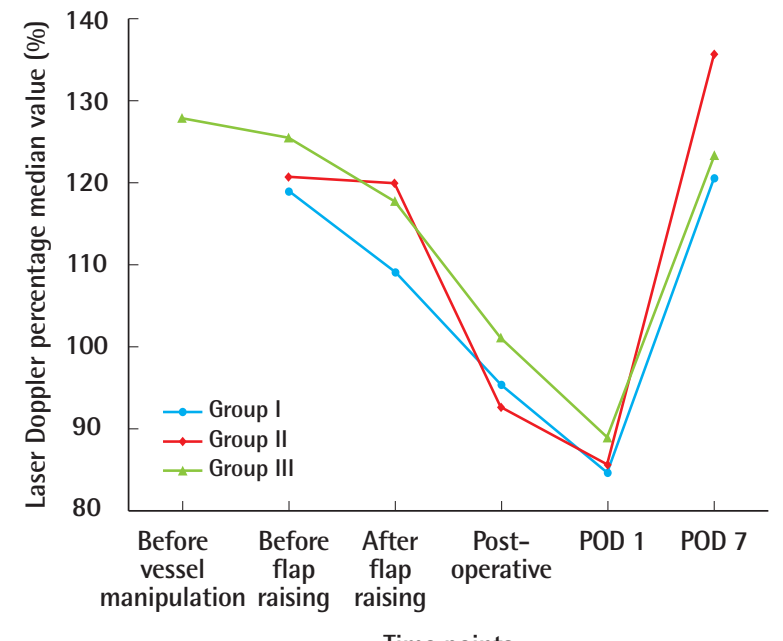

Time points
Fig. 5. Laser Doppler flowmetry: results for P3

Median laser Doppler flowmetry results for P3 at different time points. Group I (control), group II (acute inflow optimization), and group III (arterial preconditioning). POD, postoperative day.

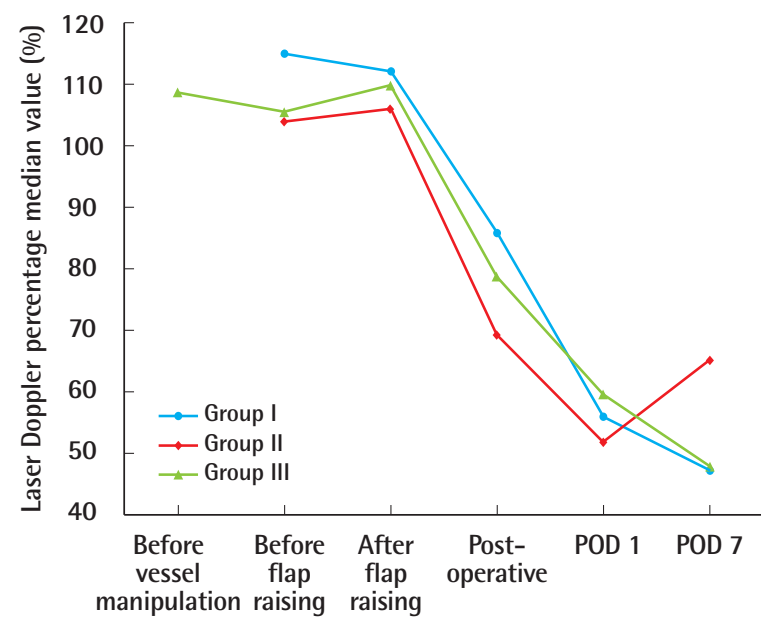

Time points in the control group $(\mathrm{P}=0.004)$ and the delayed inflow optimization group $(\mathrm{P}=0.01)$.

\section{DISCUSSION}

A perforator flap is defined as a cutaneous and subcutaneous tissue area nourished by a perforating vascular branch originating from an axial vessel and passing through certain structural elements of the body, besides interstitial connective tissue and fat, before reaching the skin [1]. In recent years, major efforts have been made to conduct anatomical studies of skin vascularization [14]. This constitutes the basis for the development of reconstructive techniques with low donor site morbidity, especially after oncological excision and trauma leading to a loss of substance with major functional and aesthetic implications [15]. Perforator-based flaps, either as free flaps or local pedicled flaps, have been established as a reconstructive method. However, local perforator flaps have shown a higher rate of partial necrosis than free flaps in lower extremity reconstruction [4]; therefore, clinical interest has emerged in methods aiming to increase local perforator flap survival.

Supercharging is a procedure intended to improve the arterial inflow of a vascularized flap through an additional arterial pedicle or anastomosis, while superdrainage refers to procedures performed to optimize the venous outflow of a flap (e.g., by adding another venous pedicle or anastomosis). Although vascular manipulations in rat abdominal flaps have been studied before
[16], the results have generally been conflicting. On one hand, some authors found that arterial supercharging alone or combined with venous superdrainage improved the flap survival rate compared to venous superdrainage alone or control groups $[9,17]$. On the other hand, studies have reported that venous superdrainage yielded a statistically significant improvement in flap survival when compared to control groups $[7,8,10,18,19]$.

Nonetheless, a general characteristic of all these experimental studies is the use of supplemental vascular pedicles to increase flap survival. However, clinical experience shows that partial flap necrosis is mainly a problem in pedicled perforator flaps [4], where the addition of a supplemental pedicle could be anatomically impossible or surgically undesirable.

The innovation of our experimental design consists of a different way of manipulating the vascular inflow within the perforator flap pedicle by ligating the femoral artery just distal to the perforator, thereby directing the entire femoral artery flow to the flap.

This novel approach does not require the addition of a supplemental vascular pedicle, as has been done by other researchers investigating flap supercharging; therefore, we chose to use the term "inflow optimization" to describe our approach in order to avoid confusions.

Our digital planimetry results clearly showed that optimization of arterial inflow in the flap pedicle led to increased flap survival at POD 7. We chose POD 7 as the endpoint of our study based on the extant literature on experimental flap models $[20$, 
21] and on the extensive research of Taylor et al. [22] showing that vascular delay reaches its maximum after 48-72 hours and remains this way afterwards. Our data suggest that it makes no significant difference whether optimization of arterial inflow is performed acutely or as a preconditioning strategy. Although the acute optimization strategy (group II) showed higher survival rates, the difference from group III did not reach statistical significance; therefore, we have no empirical basis upon which to make a strong recommendation regarding the timing of optimization of arterial inflow.

Preconditioning of a flap with the purpose of opening the choke vessels that exist between neighboring territories, as an attempt to capture angiosomes that would otherwise be lost, has been clinically and experimentally investigated in models of flap delay $[21,22]$. Classic delay refers to a flap border incision, while strategic or vascular delay is defined as the specific ligation of vessels before flap elevation [23]. Although we do use selective, specific vessel ligation, we do not induce controlled ischemia in the flap region as is done in classical flap delay. Therefore, we consider our model not to be a flap delay model, but an alternative way to optimize blood flow in the pedicle, acutely or as a preconditioning strategy.

According to the angiographic studies conducted by Coskunfirat et al. [12] in this same flap model, the three points we marked on the flaps corresponded to the center of three separate angiosomes: the first one (P1) supplied by the musculocutaneous perforator arising from the femoral artery, the second one (P2) supplied by the deep circumflex iliac artery perforator, and the third one (P3) receiving its blood supply from the intercostal arteries.

For P2, corresponding to the second angiosome, the lowest flow values in all flaps were encountered on POD 1, after which the flow increased in all groups. Taylor et al. [22] recorded a spasm of the choke vessels immediately after the operation. Afterwards, these vessels continued to dilate, reaching their greatest expansion 48-72 hours postoperatively. By POD 7, the enlargement of the choke vessels reached a plateau that was maintained. Our study revealed the same phenomenon, but was quantified by means of laser Doppler flowmetry, with a statistically significant increase in the flow between POD 1 and POD 7 in all groups.

In the third angiosome (P3), all groups showed a statistically significant decrease in flow between the first measurement and POD 7. This result was anticipated, since this phenomenon has already been described in the literature [12], but the inflow optimization group had statistically significantly higher values than the other groups. This result could be accounted for by the increase in flow between POD 1 and POD 7.
Our study clearly shows that inflow optimization in the flap pedicle had significant effects on flap survival in a musculocutaneous perforator flap model in rats. Although the laser Doppler data suggest involvement of the complex choke vessel system, the mechanism underlying these changes remains largely unknown.

Our experimental work naturally has some limitations; for instance, it did not investigate the role of venous drainage in flap survival. Even though manipulation of the femoral vein (i.e., ligation of the vein distal to the perforator) is possible, this procedure would eliminate the Venturi effect, which has been shown to make a large contribution to blood flow from the perforating to the deep veins $[24,25]$ and therefore might actually reduce venous flow. Furthermore, in our experiment we did not observe venous congestion in any flaps, suggesting that the increased blood flow in the flap can be readily drained by the pedicle veins. However, it is clear that more extensive studies of this perforator flap model are necessary to definitively address the role of venous drainage. Another shortcoming of our study is the lack of data to ascertain the mechanisms by which in-pedicle flow optimization leads to increased tissue perfusion and the observed flap survival benefits. Further studies should investigate the mechanisms of angiogenesis that are ultimately responsible for our results.

The reconstructive surgical literature has shown that perforator flaps raised as free flaps have a significantly lower rate of partial flap necrosis than local pedicled perforator flaps [4]. Our data experimentally confirm these clinical observations by showing that flaps with acute inflow optimization (group II) had significantly better survival. A free perforator flap is by definition a flap with acute inflow optimization, since all flow is directed to the flap's pedicle; therefore, our data suggest that this leads to better flap survival and the observed reduction in partial necrosis. Therefore, we propose that our data have the following implications for clinical practice regarding local pedicled perforator flaps: First, for a local pedicled perforator flap perforator, dissection should be carried out to the main emerging arteriovenous bundle and the artery should be ligated when doing so is clinically feasible (e.g., propeller flaps in the trunk, back, or partially in the thigh). Second, if the main source artery of a local pedicled perforator flap is already ligated or thrombosed distal to the perforator's emergence (e.g., a non-reconstructed arterial lesion in the lower leg with good perfusion), it is safe to assume that a flap raised on the first viable perforator would have good perfusion, thereby providing a solid indication for this technique in selected cases.

Overall, although we showed that optimization of arterial flow in the pedicle of a musculocutaneous perforator flap in a rat 
model led to increased flap survival regardless of whether it was performed acutely or as a preconditioning strategy, it should be noted that these are preliminary results. Although they could have clinical implications, further characterization of the mechanisms involved in improved flap survival should be first addressed.

\section{NOTES}

\section{Conflict of interest}

No potential conflict of interest relevant to this article was reported.

\section{Ethical approval}

The study was approved by the Ethics Committee for Animal Experimentation, Bern, Switzerland (approval No. 89/16) and treated according to the Public Health Service Policy on $\mathrm{Hu}-$ mane Care and Use of Laboratory Animals during the entire experiment.

\section{Author contribution}

Conceptualization: R Olariu, D Sabau, AV Georgescu, MA Constantinescu. Data curation: R Olariu, HL Moser, I Lese. Formal analysis: R Olariu, HL Moser, I Lese, AO Grobbelaar. Funding acquisition: R Olariu, MA Constantinescu. Methodology: R Olariu, HL Moser, D Sabau, AV Georgescu. Project administration: R Olariu, MA Constantinescu. Visualization: R Olariu, I Lese, HL Moser, AO Grobbelaar. Writing - original draft: I Lese, R Olariu. Writing - review \& editing: R Olariu, I Lese, AO Grobbelaar, D Sabau, AV Georgescu, MA Constantinescu. Approval of final manuscript: all authors.

\section{ORCID}

\begin{tabular}{|c|c|}
\hline Radu Olariu & https://orcid.org/0000-0002-0204-4432 \\
\hline Helen Laura Moser & https://orcid.org/0000-0002-8379-4760 \\
\hline Ioana Lese & https://orcid.org/0000-0002-3007-097X \\
\hline Dan Sabau & https://orcid.org/0000-0002-6163-3974 \\
\hline \multicolumn{2}{|c|}{ Alexandru Valentin Georgescu } \\
\hline & https://orcid.org/0000-0003-1233-2742 \\
\hline \multicolumn{2}{|c|}{ Adriaan Ockert Grobbelaar } \\
\hline & https://orcid.org/0000-0003-2001-5173 \\
\hline
\end{tabular}

https://orcid.org/0000-0001-9188-4785

\section{REFERENCES}

1. Blondeel PN, Van Landuyt KH, Monstrey SJ, et al. The "Gent" consensus on perforator flap terminology: prelimi- nary definitions. Plast Reconstr Surg 2003;112:1378-83.

2. Koshima I, Soeda S. Inferior epigastric artery skin flaps without rectus abdominis muscle. BrJ Plast Surg 1989;42:645-8.

3. Quaba O, Quaba A. Pedicled perforator flaps for the lower limb. Semin Plast Surg 2006;20:103-11.

4. Bekara F, Herlin C, Somda S, et al. Free versus perforatorpedicled propeller flaps in lower extremity reconstruction: what is the safest coverage? A meta-analysis. Microsurgery 2018;38:109-19.

5. Bekara F, Herlin C, Mojallal A, et al. A systematic review and meta-analysis of perforator-pedicled propeller flaps in lower extremity defects: identification of risk factors for complications. Plast Reconstr Surg 2016;137:314-31.

6. Tanaka Y, Tajima S. The influence of arterial inflow and venous outflow on the survival of reversed-flow island flaps: an experimental study. Plast Reconstr Surg 1997;99:2021-9.

7. Hallock GG, Rice DC. Efficacy of venous supercharging of the deep inferior epigastric perforator flap in a rat model. Plast Reconstr Surg 2005; 116:551-5.

8. Groth AK, Campos AC, Goncalves CG, et al. Effects of venous supercharging in deep inferior epigastric artery perforator flap. Acta Cir Bras 2007;22:474-8.

9. Yamamoto $Y$, Sakurai $H$, Nakazawa $H$, et al. Effect of vascular augmentation on the haemodynamics and survival area in a rat abdominal perforator flap model. J Plast Reconstr Aesthet Surg 2009;62:244-9.

10. Gumus N, Erkan M, Ercocen AR. Vascular pressure monitorization for necessity of vascular augmentation in a rat extended abdominal perforator flap model. Microsurgery 2012;32:303-8.

11. Georgescu AV, Matei I, Ardelean F, et al. Microsurgical nonmicrovascular flaps in forearm and hand reconstruction. Microsurgery 2007;27:384-94.

12. Coskunfirat OK, Islamoglu K, Ozgentas HE. Posterior thigh perforator-based flap: a new experimental model in rats. Ann Plast Surg 2002;48:286-91.

13. Tim CR, Martignago CCS, da Silva VR, et al. A comparison of three methods for the analysis of skin flap viability: reliability and validity. Adv Wound Care (New Rochelle) 2018; 7:157-63.

14. Saint-Cyr M, Wong C, Schaverien M, et al. The perforasome theory: vascular anatomy and clinical implications. Plast Reconstr Surg 2009; 124:1529-44.

15. Artiaco S, Battiston B, Colzani G, et al. Perforator based propeller flaps in limb reconstructive surgery: clinical application and literature review. Biomed Res Int 2014;2014:690649.

16. Chang H, Nobuaki I, Minabe T, et al. Comparison of three different supercharging procedures in a rat skin flap model. 
Plast Reconstr Surg 2004;113:277-83.

17. Chen W, Li YQ, Tang Y, et al. Which vessel, the artery or the vein is more important in vascular supercharge: an investigation of vascular changes on rat abdominal supercharging flap models. Zhonghua Zheng Xing Wai Ke Za Zhi 2013;29:40-4.

18. Sano K, Hallock GG, Rice DC. Venous "supercharging" augments survival of the delayed rat TRAM flap. Ann Plast Surg 2003;51:398-402.

19. Zheng J, Xi S, Ding M, et al. Effects of venous superdrainage and arterial supercharging on dorsal perforator flap in a rat model. PLoS One 2016;11:e0160942.

20. Dunn RM, Mancoll J. Flap models in the rat: a review and reappraisal. Plast Reconstr Surg 1992;90:319-28.
21. Ghali S, Butler PE, Tepper OM, et al. Vascular delay revisited. Plast Reconstr Surg 2007;119:1735-44.

22. Taylor GI, Corlett RJ, Ashton MW. The functional angiosome: clinical implications of the anatomical concept. Plast Reconstr Surg 2017;140:721-33.

23. Seyhan T, Deniz M, Borman H, et al. Comparison of two different vascular delay methods in a rat cranial epigastric perforator flap model. Ann Plast Surg 2010;64:89-92.

24. Gianesini S, Sisini F, Di Domenico G, et al. Lower limbs venous kinetics and consequent impact on drainage direction. Phlebology 2018;33:107-14.

25. Uhl JF, Gillot C. Anatomy of the veno-muscular pumps of the lower limb. Phlebology 2015;30:180-93. 